\title{
The interaction between surface color and color knowledge: Behavioral and electrophysiological evidence
}

\author{
Inês Bramão a,b,c , Luís Faísca a , Christian Forkstam ${ }^{\text {a,b,c }}$, Filomena Inácio a , Susana Araújo a,b,c \\ Karl Magnus Petersson ${ }^{\mathrm{a}, \mathrm{b}, \mathrm{c}}$, Alexandra Reis ${ }^{\mathrm{a}, *}$ \\ ${ }^{a}$ Cognitive Neuroscience Research Group, Deparmento de Psicologia, Faculdade de Ciências Humanas e Sociais, Institute of Biotechnology E Bioengineering/CBME, Universidade \\ do Algarve, Faro, Portugal \\ ${ }^{\mathrm{b}}$ Max Planck Institute for Psycholinguistics, Nijmegen, The Netherlands \\ ${ }^{\mathrm{c}}$ Donders Institute for Brain, Cognition, and Behaviour, Radboud University Nijmegen, The Netherlands
}

\section{A R T I C L E I N F O}

\section{Article history:}

Accepted 13 October 2011

Available online 8 November 2011

\section{Keywords:}

ERPs

Object identification

Surface color

Color knowledge

Color verification

\begin{abstract}
A B S T R A C T
In this study, we used event-related potentials (ERPs) to evaluate the contribution of surface color and color knowledge information in object identification. We constructed two color-object verification tasks - a surface and a knowledge verification task - using high color diagnostic objects; both typical and atypical color versions of the same object were presented. Continuous electroencephalogram was recorded from 26 subjects. A cluster randomization procedure was used to explore the differences between typical and atypical color objects in each task. In the color knowledge task, we found two significant clusters that were consistent with the N350 and late positive complex (LPC) effects. Atypical color objects elicited more negative ERPs compared to typical color objects. The color effect found in the N350 time window suggests that surface color is an important cue that facilitates the selection of a stored object representation from long-term memory. Moreover, the observed LPC effect suggests that surface color activates associated semantic knowledge about the object, including color knowledge representations. We did not find any significant differences between typical and atypical color objects in the surface color verification task, which indicates that there is little contribution of color knowledge to resolve the surface color verification. Our main results suggest that surface color is an important visual cue that triggers color knowledge, thereby facilitating object identification.
\end{abstract}

(c) 2011 Elsevier Inc. All rights reserved.

\section{Introduction}

Perceiving that a strawberry is red versus knowing and recalling that a strawberry is red are distinct cognitive operations. The surface color of an object can be defined as the percept generated by the color present in the object image (e.g., the color red in a picture of a red strawberry), whereas color knowledge refers to the knowledge about the prototypical color of an object and is represented and stored together with other semantic information about the object (e.g., the knowledge that strawberries are typically red). Tanaka and collaborators (2001) proposed the "Shape + Surface" model of object recognition which makes a distinction between surface color and color knowledge. According to this model, object recognition is jointly determined by bottom-up influences (surface color) and top-down influences (color knowledge). In this context,

\footnotetext{
* Corresponding author. Address: Departamento de Psicologia, Faculdade de Ciências Humanas e Sociais, Campus de Gambelas, Universidade do Algarve, 8005-139 Faro, Portugal. Fax: +351 289819403.

E-mail address: aireis@ualg.pt (A. Reis).
}

one can ask how these two representations interact during object recognition. It is well documented that surface color contributes to the recognition of diagnostic color objects (for a review, see Tanaka et al., 2001). For surface color to be a relevant cue in object recognition, cognitive processing must identify whether the color present in the object is, or is not, appropriate for a given object. For this to occur, semantic color knowledge must be accessed. Following this reasoning, one can hypothesize that when semantic color knowledge is activated together with other visual and functional object properties, recognition is faster and more accurate.

To study how surface color and color knowledge might interact during object recognition, Joseph and collaborators (Joseph, 1997; Joseph \& Proffitt, 1996) manipulated the input color independently of color knowledge in a series of verification tasks. The authors found that color knowledge significantly influenced object recognition; for example, an image of a purple apple was more likely to be mistaken for a cherry than for a blueberry. This interference effect occurs because both apples and cherries are typically red and not because the apple was colored purple, the typical color of blueberries. The same pattern of results was obtained when uncolored 
pictures were used, suggesting that the conceptual processing of color did not depend on the presence of surface color. In their verification tasks participants were asked to verify an object target against three types of distracters: similar in color and shape; dissimilar in color and similar in shape; and a distracter dissimilar in color and shape. In a recent study, we used a similar object verification task in which the effect of color information was assessed independently of the effects of shape information, by adding a fourth distracter similar in color and dissimilar in shape in a $2 \times 2$ factorial design (Bramão, Faísca, Petersson, \& Reis, 2010). Since the object recognition system is a shape-driven system (Tanaka et al., 2001), it is important to experimentally manipulate the effects of color independently of the effects of shape. We observed an interference of color knowledge on object verification when the color knowledge, activated by a previously presented object name (e.g., orange), were consistent with surface color information provided by an object picture (e.g., carrot). This interference effect was strongly dependent on surface color. When the objects were presented in black and white or in an atypical color, the interference effect disappeared. This finding suggests that the appropriate surface color input promotes the activation of stored color knowledge in the cognitive system (Bramão et al., 2010).

One approach to investigate these processes in the time domain is to characterize the underlying neural processing with event-related potentials (ERPs). Previous ERP studies have identified two different time windows associated with object identification. The first observed difference between successful and non-successful recognition starts around $250 \mathrm{~ms}$ after stimulus onset and is characterized by a frontal negativity peaking around $350 \mathrm{~ms}$ (N350). The N350 is more negative when the objects are more difficult to recognize or not recognized at all. It has been hypothesized that the N350 reflects the selection of a long-term memory representation - a stored structural description - that best matches the input image (Pietrowsky et al., 1996; Schendan \& Kutas, 2002, 2003, 2007). The second observed difference starts around $550 \mathrm{~ms}$ after stimulus onset and is characterized by a broadly distributed late positive complex (LPC). The LPC is also more negative for non-recognized compared to recognized objects. Modulation of the LPC has been linked to object identification and is hypothesized to reflect the activation of semantic knowledge associated with the object, including the object name (Mazerolle, D'Arcy, Marchand, \& Bolster, 2007; Pietrowsky et al., 1996; Schendan \& Kutas, 2002, 2003, 2007; Stuss, Picton, Cerri, Leech, \& Stethem, 1992). An additional effect, which reflects semantic knowledge integration and/or retrieval is the N400 effect, which was initially related to words that are semantically unrelated or unusual in a given semantic sentence context (Kutas \& Hillyard, 1980a, 1980b). The N400 component is characterized by a negativity peaking around $400 \mathrm{~ms}$ after stimulus onset (Barrett \& Rugg, 1990; Ganis, Kutas, \& Sereno, 1996; Hamm, Johnson, \& Kirk, 2002; Holcomb \& McPherson, 1994; McPherson \& Holcomb, 1999; Nigam, Hoffman, \& Simons, 1992; Pietrowsky et al., 1996; Pratarelli, 1994; Stuss et al., 1992) and was first described for pictures by Barrett and Rugg (1990). Barrett and Rugg (1990) reported that pictures that were semantically unrelated to a previous priming stimulus elicit a greater negative ERP component around $400 \mathrm{~ms}$ after stimulus onset, as compared to pictures that were semantically related to a previous primer.

In this study, we recorded ERPs to investigate how surface color and color knowledge interact during object identification. Previous behavioral findings have shown that surface color information triggers the activation of stored color knowledge (Bramão et al., 2010). If this is the case, then when objects are presented in the appropriate color, color knowledge should be activated more rapidly than when objects are presented in an inappropriate color. To evaluate this hypothesis, we constructed a color knowledge verification task with high color diagnostic objects, that is, objects that are strongly associated with a prototypical color (Tanaka \& Presnell, 1999). Participants were instructed to verify whether the prototypical color of the presented object matched a previously presented color name. The actual object color was manipulated in order to evaluate the contribution of surface color to verification, by presenting objects in both typical and atypical color (see Fig. 1). We predicted ERP differences between typically and atypically colored objects in the ERP components previously identified as being involved in object identification. We assessed the role of surface color in the activation of stored color knowledge by comparing atypical versus typical color objects. If the surface color modulates the retrieval of color knowledge, atypical color objects should elicit a more negative ERPs in association with the N350 and LPC components. Furthermore, we also explored the differences between matching and non-matching trials. The non-matching condition creates incongruence between the color name and the color knowledge activated by a given object. Thus, we expected that non-matching trials were associated with more negative ERPs related to the N400 component, considering the previous findings associating this component with incongruent semantic contexts.

We also evaluated the contribution of color knowledge in the surface color verification task. We have previously observed that color knowledge is not automatically activated in the absence of surface color input (Bramão et al., 2010). Given the fact that stored color knowledge is not necessary to resolve the surface color verification task, we predicted that this information would only be activated to a modest degree, if at all. To investigate this hypothesis, a surface color verification task was designed in which high color diagnostic objects were used. The actual color of the objects was manipulated in order to evaluate the contribution of color knowledge to surface color verification. To that end, we presented both typical and atypical color versions of the same object (see Fig. 1). Participants were instructed to verify whether the surface color of the object matched a previously presented color name. If color knowledge is automatically activated during the surface color verification task, differences in the behavioral and in the electrophysiological results should emerge when comparing atypical and typical color conditions, and when comparing non-matching and matching trials in the ERPs components previously related to successful object identification.

\section{Methods}

\subsection{Participants}

Twenty-six right-handed Portuguese native speakers (mean age $( \pm$ std $)=23 \pm 4$ years, range 18 -32 years; mean years of education $( \pm$ std $)=14 \pm 2$ years, range $12-18$ years; 9 males and 17 females $)$ with normal or corrected-to-normal vision participated in the study. All subjects completed health questionnaires, and none indicated a history of head injury or other neurological or psychiatric problems. All subjects read and signed an informed consent form describing the procedures, which adhered to the guidelines set out by the Declaration of Helsinki. The study was approved by the local ethics committee.

\subsection{Stimulus material}

We used eight colors in the experiment (red, gray, orange, green, yellow, brown, pink and white). Each of the colors was easily distinguishable from the others. We selected 56 black and white line drawings from the picture database at the Max Planck Institute. The drawings in this database are based on the Snodgrass and Vandervart (1980) set. We selected objects strongly associated 


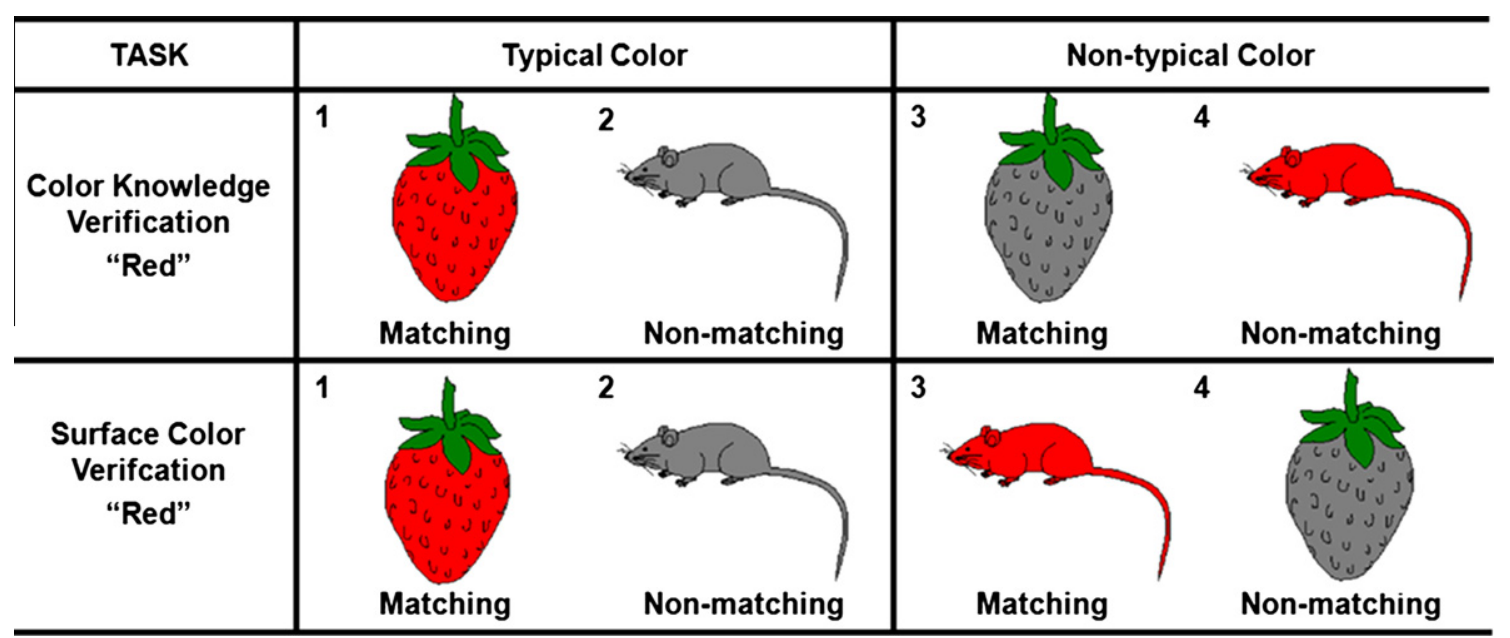

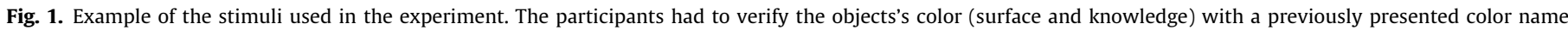
"red". (For interpretation of the references to color in this figure legend, the reader is referred to the web version of this article.)

with one of selected colors based on the color-diagnosticity scores of Rossion and Pourtois (2004; where, on the original scale, 1 means 'the color of the object depicted is not diagnostic at all, i.e., this object could be in any other color equally well' and 5 means 'the color depicted is highly diagnostic of the object, i.e., the object appears only with that color in real life'; diagnostic color mean of the selected objects $( \pm$ std $)=4.4 \pm 0.4$, range $3.3-5.0)$. In order to keep color frequency constant, we selected the same number of objects for each color (seven objects strongly associated with each color, in total $8 \times 7=56$ color-object combinations, see Appendix A). Adobe Photoshop 7.0 was used to apply the proper color to the internal surface of the objects. To test only color effects, other surface features such as texture and details were removed or minimized.

An atypical color version of each object was created. To construct the atypical color version we rotated the typical colors across objects, whilst ensuring that typical and atypical colored objects were matched for color frequency and luminance. For example, the red color was used to construct the atypical color version of the typical gray objects and the gray color was used to construct the atypical color version of the typical red objects. The three other color pairs used were orange-green, yellow-brown and whitepink.

\subsection{Experimental procedures}

Two computerized verification tasks were designed: a knowledge verification task and a surface color verification task. The only difference between the two tasks was the instructions given to the subjects. Each verification task comprised 24 blocks (three blocks for each color). Each block started with the presentation of a color name followed by 28 objects ( 14 typical and 14 atypical color objects - half of them matched and the other half did not match with the given color name). The color name remained on the screen until subjects pressed a key. In total, each verification task comprised 672 trials, distributed over 24 blocks ( 28 trials per block). The trials were divided in two types: matching (336) and non-matching (336) trials, and in two color conditions: typical and atypical color (168 trials per trial type and per condition). The same object was presented three times in each color version and for each trial type (56 objects $\times 2$ color versions $\times 3$ times each $\times 2$ trial types). Both blocks and trials within blocks were presented in a randomized order. In the knowledge verification task, participants were asked to decide whether the prototypical color of the presented object matched (or not) a previously presented color name (see Fig. 1).
In the surface color verification task, participants were asked to decide whether or not the surface color of the object matched a previously presented color name, ignoring the prototypical color of the object (see Fig. 1).

Presentation 0.7 software (nbs.neuro-bs.com/presentation) was used to display the stimuli on a computer screen (size: 19"; spatial resolution: $1024 \times 768$; color resolution: 24 bits) and to register the response times. Each block lasted about $2 \mathrm{~min}$ and started with the presentation of a color name, which remained on the screen until the subject pressed a key. This was followed by 28 trials. Each trial started with a fixation cross $(+)$ presented at the center of the screen for $500 \mathrm{~ms}$, followed by presentation of the object picture ( $760 \times 550$ pixels) for $120 \mathrm{~ms}$. Participants were instructed to respond as accurately and as quickly as possibly by pressing one of the two response keys (selection of the response finger was balanced within subjects: half of the participants started with their right/left hand for yes/no responses and in the middle of each verification task the response hand changed). The trial ended with the response of the participant. Between each block, subjects rested for a few minutes (for as long as they desired). Between each task, subjects paused for at least $10 \mathrm{~min}$. The inter-stimulus interval (ISI) varied randomly between 750 and $1250 \mathrm{~ms}$. During this period, indicated by three stars $(* * *)$ on the screen, subjects were allowed to blink their eyes (Fig. 2) The subjects were instructed to fixate on the center of the screen and to avoid eye and body movements during the recording session. The task order was balanced over subjects. Before each verification task and change of response hand, subjects were allowed 16 practice trials. The entire recording procedure lasted approximately $2 \mathrm{~h}$.

\subsection{EEG recordings}

Continuous electroencephalogram (EEG) was recorded from 64 $\mathrm{Ag} / \mathrm{AgCl}$ active electrodes held in place on the scalp by an elastic cap. The electrode montage included 10 midline sites and 27 sites over each hemisphere. Two additional electrodes (CMS/DRL nearby $\mathrm{Pz}$ ) were used as an online reference (for a complete description, see biosemi.com; Schutter, Leitner, Kenemans, \& van Honk, 2006). Three other electrodes were attached over the right and left mastoids and below the right eye (to monitor eye movements and blinks). Bioelectrical signals were amplified using an ActiveTwo Biosemi amplifier (DC-100 Hz bandpass, $3 \mathrm{~dB} /$ octave) and were continuously sampled (24-bit sampling) throughout the experiment at a rate of $512 \mathrm{~Hz}$. 


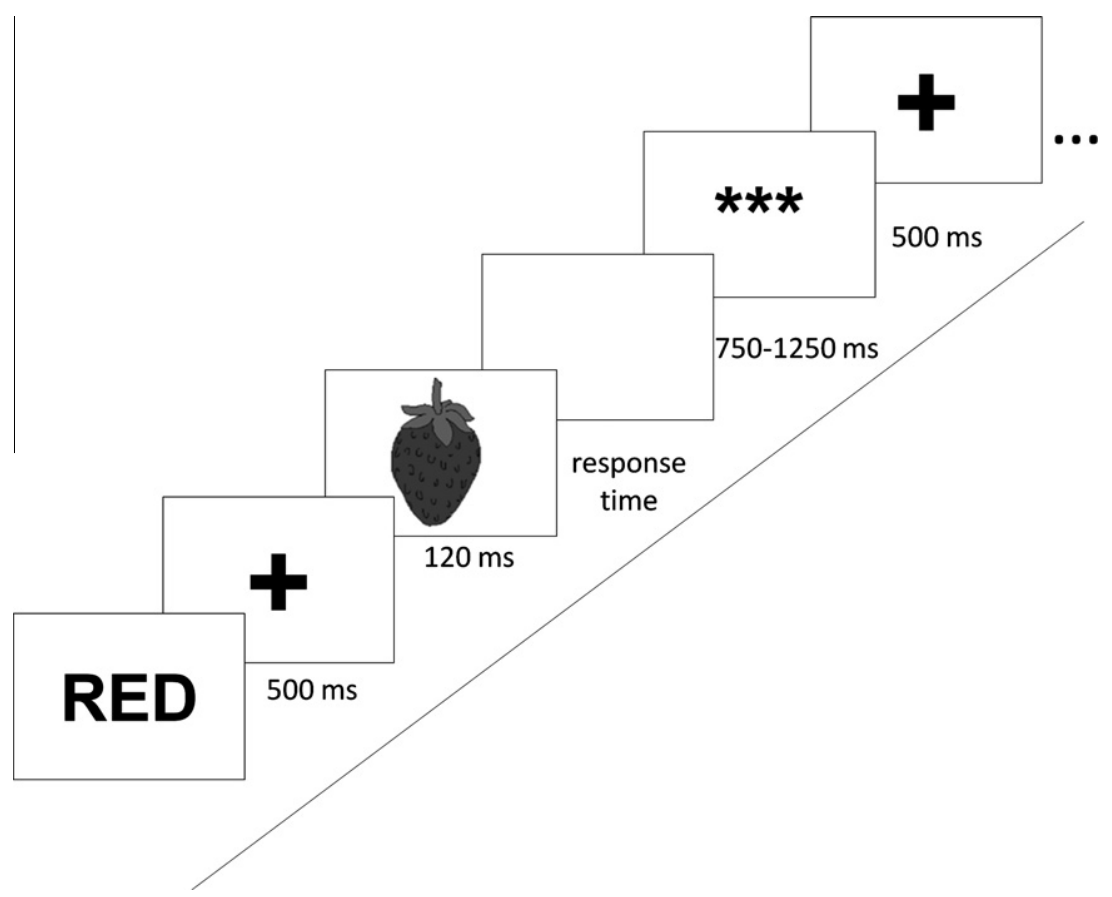

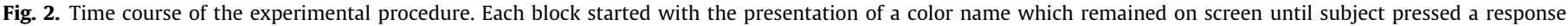
button. Each trial started with the presentation of a fixation point $(+)$, followed by the image of the object and ended with subject response.

\subsection{ERP data analysis}

The EEG data was analyzed using FieldTrip which is an open source toolbox for EEG and MEG analysis developed at the F.C. Donders Centre for Cognitive Neuroimaging (Oostenveld, Fries, \& Jensen, 2009; documentation and algorithms available at ru.nl/ fcdonders/fieldtrip). ERP data were computed using a $1000 \mathrm{~ms}$ epoch (from $200 \mathrm{~ms}$ before to $800 \mathrm{~ms}$ after the stimulus onset) that was time-locked to the onset of the stimuli. Before averaging, epochs that contained muscle and/or eye movement artifacts were visually rejected, for each subject, and discarded from the analysis. The electrodes Fp2, F7 and F8 in the electrode-cap were used to record the EOG (i.e., to detect vertical and horizontal eye movements). The vertical eye movements were captured by calculating the amplitude difference between the electrode placed under the right eye and Fp2. In the same way, the horizontal eye movements were captured by calculating the amplitude difference between F7 and F8. Incorrect response trials were also excluded as well as any trial with implausibly long/short response times (every trial with a latency 2.5 standard deviations away from the mean for each participant and condition was excluded from further analysis). After artifact rejection and corrections, an average of 425.5 trials (63.3\%) per subject, from the total set of 672 , entered the final analysis of the color knowledge verification task. The ERP averages were based on 109.1 (25.6\%) matching typical color trials, 104.5 (24.6\%) matching atypical color trials, 107.2 (25.2\%) non-matching typical color trials and 104.7 (24.6\%) non-matching atypical color trials. For the surface color verification task, the final analysis was based upon an average of 442.2 trials (65.8\%) per subject, from the total set of 672 . The ERP averages were based on 109.9 (24.9\%) matching typical color trials, 109.6 (24.8\%) matching atypical color trials, 111.3 (25.1\%) non-matching typical color trials, 111.4 (25.2\%) non-matching atypical color trials. All trials included in the final analysis were filtered, using a low-pass filter of $30 \mathrm{~Hz}$ and a high-pass filter of $0.01 \mathrm{~Hz}$ and transformed to an average reference (eye electrodes were excluded to compute the common reference). The $200 \mathrm{~ms}$ prior to the stimulus onset served as the baseline for the amplitude measurement for each channel.
Separate ERP grand-averages were calculated for each experimental condition.

To investigate the contribution of surface color to color knowledge verification as well as the contribution of color knowledge to surface color verification, we explored the differences between the ERPs grand-averages elicited by typical and atypical color objects and by matching and non-matching trials (between 100 and $800 \mathrm{~ms}$ after stimulus onset) in each task using cluster randomization analyses. The cluster randomization method that Fieldtrip uses is an improved version of the method described in Maris (2004) (Maris \& Oostenveld, 2007). This test effectively controls the Type- 1 error rate in a situation involving multiple comparisons (i.e., 64 electrodes $\times 360$ time points). Briefly, the method works as follows: In a first step, all pairs (electrode, time point) are identified for which the $t$-statistics for the difference between conditions (e.g., atypical versus typical color) exceed some prior threshold. In our study, we selected the pairs whose $t$-statistics exceeded the $5 \%$ critical value of the (electrode, time)-specific $t$-statistics. The selected (electrode, time) pairs are then grouped into a number of clusters in such a way that, within every cluster, the (electrode, time) pairs form a set that is connected spatially and/or temporally. In other words, if the (electrode, time)-specific $t$-statistics that exceeded the statistical threshold were neighboring either spatially or temporally, these pairs were then grouped together as a cluster. Each cluster is assigned a cluster-level test statistic whose value equals the sum of the (electrode, time)-specific test statistics. Thus, the cluster-level test statistic depends on both the extent of the cluster and the size of the (electrode, time)-specific $t$-statistics that belong to this cluster. The Type-I error rate for the complete spatiotemporal data matrix is controlled by evaluating the cluster-level test statistic under the randomization null distribution of the maximum cluster-level test statistic. This randomization null distribution is obtained by randomizing the order of the data (e.g., atypical and typical color trials) within every participant. By creating a reference distribution from 4000 random draws, the $p$-value maybe estimated by the proportion from this randomization null distribution in which the maximum cluster-level test statistic exceeds the observed cluster level test statistic 
(this proportion is called a Monte Carlo $p$-value in the statistics literature). With this number of 4000 random draws, our Monte Carlo $p$-value is an accurate estimate of the true $p$-value. In brief, the cluster randomization $p$-value denotes the chance that such a large summed cluster-level statistic will be observed when there is actually no effect. In this way, significant clusters extending both over time and over electrodes can be identified, providing a measure both of the timing and of the distribution of the effect.

\subsection{Response time analysis}

The response times were analyzed by subject (F1) and by stimulus (F2). Response time was defined as the interval between the onset of the object picture and the subject's button press. A minimum $F(\min F)$ was calculated from the $\mathrm{F} 1$ and $\mathrm{F} 2$ analyses. This approach ensures that the results generalize over both subject- and stimulus domains (Clark, 1973; Raaijmakers, 2003; Raaijmakers, Schrijnemakers, \& Gremmen, 1999). None of the main effects or interactions that failed to reach significance in the $\min F$ calculation are reported. Overall, the participants were able to correctly verify almost all stimuli and we focused our analysis on the verification times for the correct trials with latencies within 2.5 standard deviations of the mean for each participant and condition. We excluded verification times of incorrect responses as well as implausibly long or short verification times from the analysis. In total, $11.7 \%$ of the trials were excluded (7.4\% incorrect and $4.3 \%$ excessively long/short response time). Verification times were analyzed with a repeated-measures ANOVA including task (surface/ knowledge task), color (typical/atypical) and matching (matching/non-matching) as within-subject/stimulus factors.

\section{Results}

\subsection{Behavioral results}

The results showed a significant task effect $(\min F(1,32)=178$, $P<.001$ ) - subjects responded faster in the surface verification task compared with the knowledge verification task; a main color effect was observed $(\min F(1,70)=63, P<.001)$ - subjects responded faster to typical color objects as compared to atypical color objects; and finally also a main significant effect of matching was also observed $(\min F(1,51)=130, P<.001)$ - subjects responded fast to matching trials then to non-matching trials. The two-way interaction between task and color was significant $(\min F(1,62)=53$, $P<.001)$. A Tukey HSD post hoc comparison for the subject analysis showed that in the knowledge verification task subjects were faster in responding to the typical as compared to the atypical color presentation $(P<.001)$; however, in the surface task, subjects respond equally fast to typical and atypical color presentations $(P=.95)$. The two-way interaction between color and matching was also significant $(\min F(1,63)=15, P<.001)$. A Tukey HSD post hoc comparison for the subject analysis showed that when the trials were matching the difference between typical color and atypical color was bigger $(P<.001)$ than when the trials were non-matching $(P=.005)$. The three-way interaction was also significant $(\min F(1,71)=11, P=.002$; see Fig. 3$)$. A Tukey HSD post hoc comparison for the subject analysis showed that in the surface verification task subjects performed equally fast the task independently of the color presentation, both in the matching and in the non-matching trials $(P=.99)$; In the color knowledge verification task, subjects were faster performing color verifications in the typical color version; however the advantage of color presentation was bigger for the matching trials $(P<.001)$ compared to the non-matching trials $(P=.01)$.

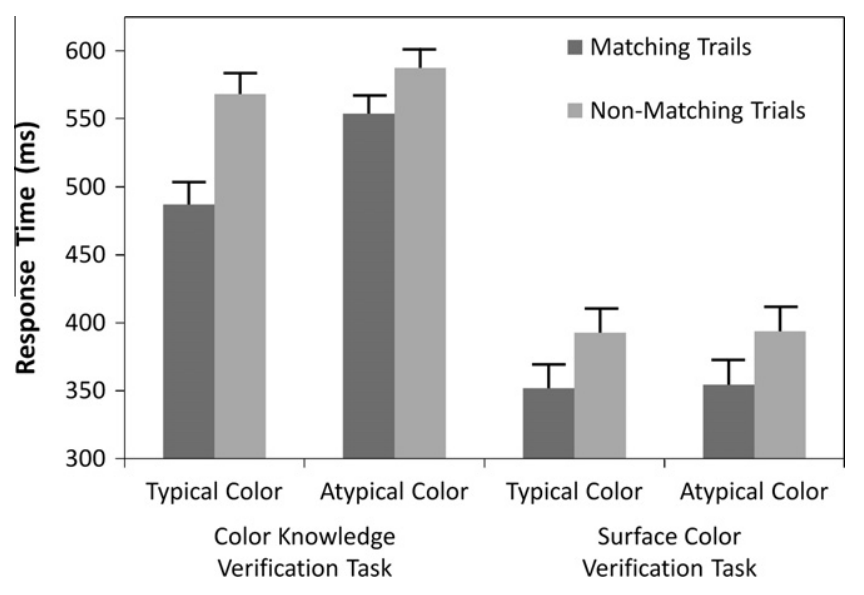

Fig. 3. Three-way interaction between the factors task, color and matching factors $(\min F(1,71)=11, P=.002)$. Error bars represent the standard error.

\subsection{Electrophysiological results}

\subsubsection{Color knowledge verification task}

The color effect was explored through the contrast between atypical and typical color objects, in both the matching and nonmatching trials. In the matching trials, the color effect was associated with a greater average negative potential over 26 anterior electrodes in a time window of 300-500 ms post-onset of the stimulus, consistent with an N350 effect (sum-T=-6309.8; $P<.001$ ), with a corresponding positive effect occurring over 20 posterior channels (sum- $T=3204.6$; $P=.001$; Fig. 4 A).

In the non-matching trials, the N350-like effect was observed in two different clusters, showing that atypical color objects were associated with a frontocentral potential of greater negativity (Fig. 4B). The first cluster was found in a time window of $260-320 \mathrm{~ms}$ after stimulus onset over 22 frontal electrodes (sum- $T=-1337.4$; $P=.005$ ), with a corresponding positive effect observed in 18 posterior channels ( sum- $T=479.3 ; P=.05$ ). Additionally, a second significant cluster was found, also consistent with the N350-like effect, over 13 frontal right channels (sum- $T=-1021.6 ; P=.01$ ) between 380 and $490 \mathrm{~ms}$ after stimulus onset. Finally, around 580-720 ms after stimulus onset, we observed that atypical color objects once more induced higher negativity in one cluster (sum- $T=-1570.2$; $P=.004$ ) over 10 right frontal channels, consistent with the LPC effect (Fig. 4B).

To explore the difference between non-matching and matching trials, we compared these two trial types with regard to typical and atypical color objects. The cluster randomization analysis identified that the non-matching trials were associated with greater central negativity compared with the matching trials for both the typical and atypical color objects in a time window of 350-600 ms postonset of the stimulus, consistent with an N400-like effect. In the typical color objects, the non-matching trials were associated with more negative amplitudes over 29 central electrodes (sum$T=-7804.5 ; P<.001)$ compared with the matching trails, with a corresponding positive effect occurring over 27 outer channels (sum- $T=3204.6 ; P=.0025$; Fig. 5 A). For the atypical color objects, the non-matching trials were associated with more negative amplitudes compared with the matching trials over 19 channels (sum- $T=-5893.7 ; P<.001$ ), with the corresponding positive effect occurring over 12 outer channels (sum- $T=2016.3 ; P=0.006$; Fig. 5B).

\subsubsection{Surface color verification task}

In this task, we did not observe any significant difference between atypical and typical color objects in either the matching (Fig. 6A) or the non-matching trials (Fig. 6B). 


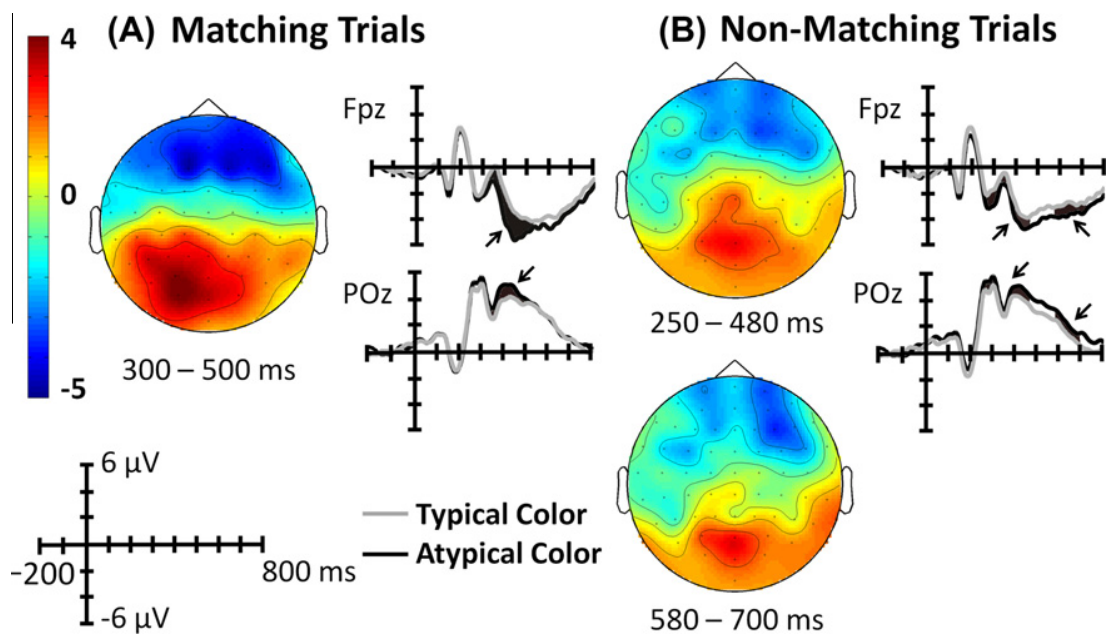

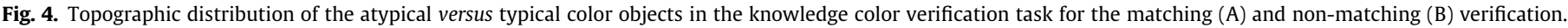

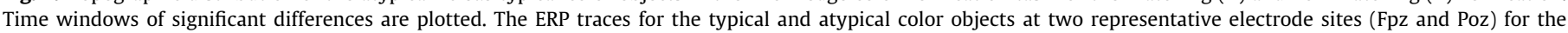

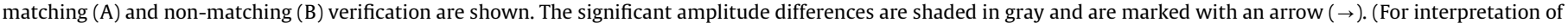
the references to color in this figure legend, the reader is referred to the web version of this article.)

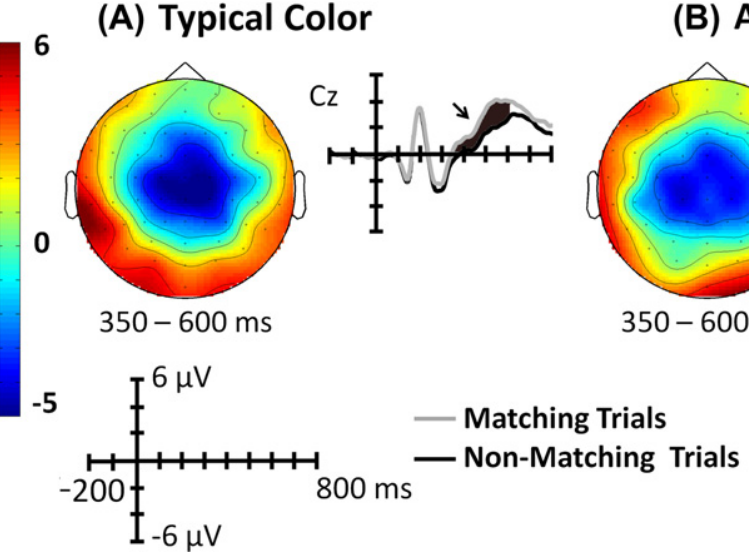

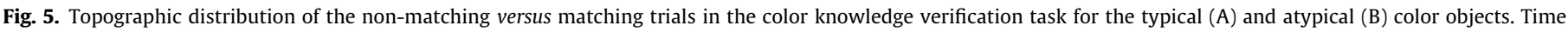

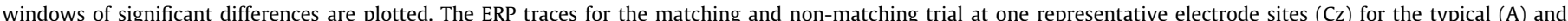

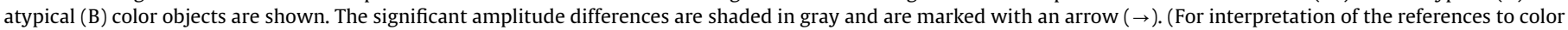
in this figure legend, the reader is referred to the web version of this article.)

To investigate the matching effect in the surface color verification task, we compared the non-matching trials against the matching ones for both typical and atypical color objects. For the typical color objects, the non-matching trials were associated with greater frontal negativity compared with the matching trials in a time window of $215-280 \mathrm{~ms}$ post-onset of the stimulus, over 26 channels (sum- $T=-2499.8 ; P<.001$ ). We observed a corresponding positive effect over 15 posterior channels (sum- $T=893.7 ; P=.006$; Fig. 7A). A second significant cluster associating the non-matching trials with greater central negativity was found over 18 central channels (sum- $T=-3004.8 ; P<.001$ ), with a corresponding positive effect occurring in 13 outer channels (sum- $T=603.3 ; P=.016$; Fig. $7 \mathrm{~A}$ ), between 315 and $480 \mathrm{~ms}$ after stimulus onset. Similar results were found for the atypical color objects. The cluster analysis also identified two significant clusters showing that the non-matching responses were associated with greater frontal-central negativity, compared with the matching responses. The first cluster was found over 27 frontal channels (sum- $T=-2.243 .3 ; P=.0075$; Fig. 7B) between 210 and $280 \mathrm{~ms}$ post-onset of the stimulus and the second cluster identified 10 central electrodes ( $\operatorname{sum}-T=-606.6 ; P=.031$; Fig. 7B) between 320 and $370 \mathrm{~ms}$ post-onset of the stimulus.

\section{Discussion}

In this study, we used event-related potentials (ERP) to better understand the contribution of color perception and stored color knowledge to object identification. We designed two color-object verification tasks - a surface color and a color knowledge verification task - where both typical and atypical color versions of the same high color diagnostic objects were presented. In the color knowledge task, the subjects were asked to verify whether or not the prototypical color of the presented objects matched with a previously presented color name, independently of the actual color of the presented object. The object color was manipulated in order to evaluate the contribution and the interference of surface color information during object identification. In the surface color verification task, subjects were asked to verify whether or not the color of the presented object matched with a previously presented color name, independently of the prototypical color of the object. The actual object color was manipulated in order to evaluate the contribution and the interference of stored color knowledge with regard to the surface color task.

Our results showed that the atypical color objects were associated with significant N350 and LPC effects in the knowledge color 
(A) Matching Trials

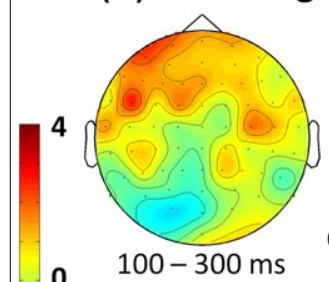

0
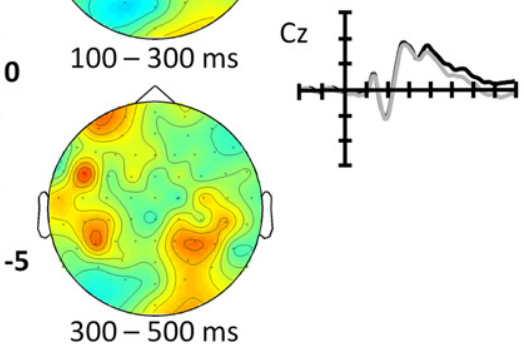

$300-500 \mathrm{~ms}$

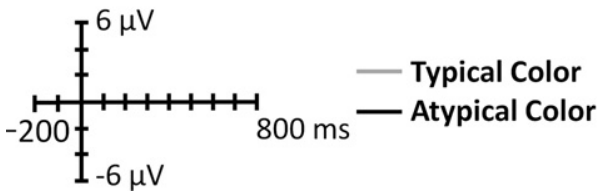

(B) Non-Matching Trials
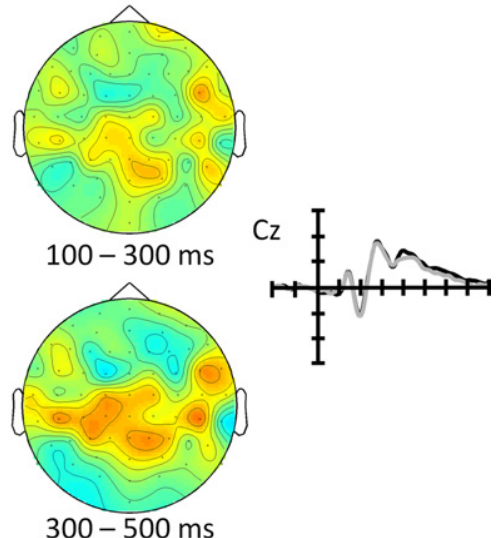

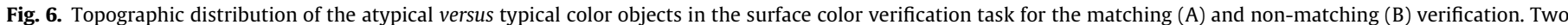

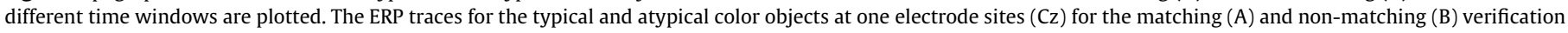
are shown. (For interpretation of the references to color in this figure legend, the reader is referred to the web version of this article.)

(A) Typical Color
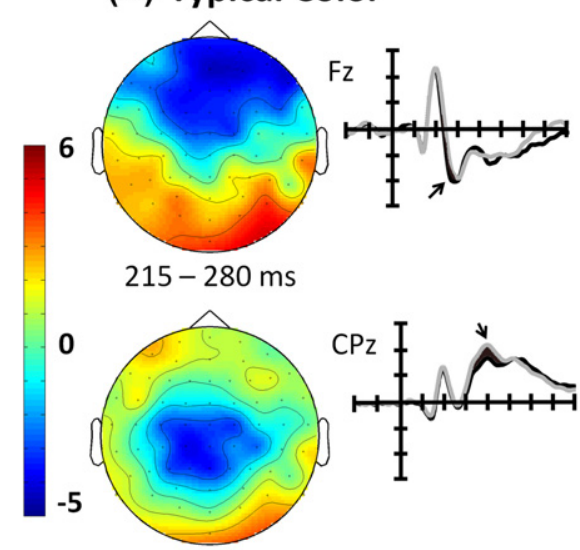

$315-480 \mathrm{~ms}$

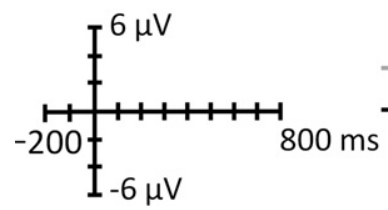

(B) Atypical Color
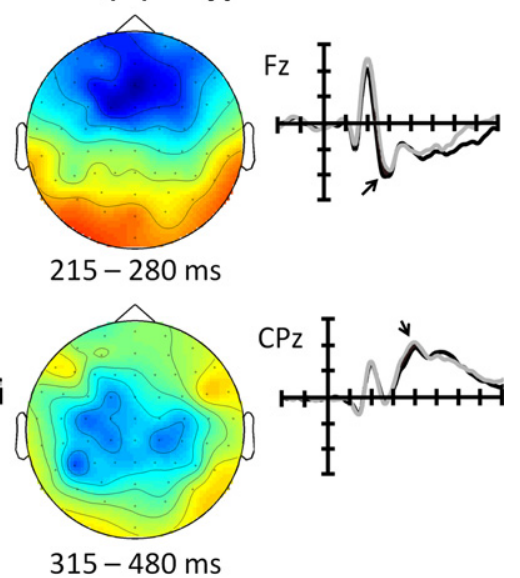

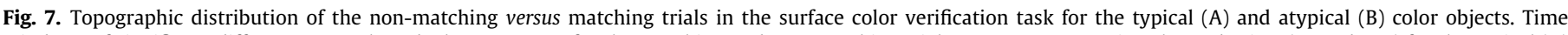

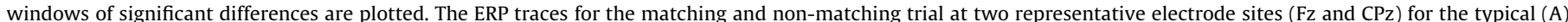

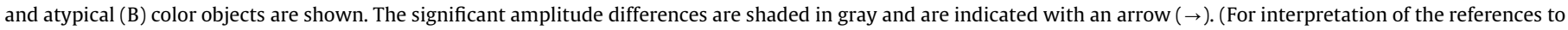
color in this figure legend, the reader is referred to the web version of this article.)

task, whereas no differences were found between the atypical and typical color conditions in the surface color task. In color knowledge verification, the differences observed between the ERP elicited by atypical and typical color objects were temporally and topographically consistent with an N350-like effect. Atypical color objects were associated with a more negative ERP over the frontal sites during the time window between 300 and $500 \mathrm{~ms}$ after stimulus onset. The N350 marks the first ERP component associated with identification success; the N350 is smaller for correctly identified compared to unidentified objects, it is sensitive to the recoverability of perceptual structure, and it is an index of the matching process between the perceptual input with a template stored in the long-term memory (McPherson \& Holcomb, 1999; Schendan \& Kutas, 2002). The visual knowledge enabling this matching ability is of generic semantic type (Schendan \& Kutas, 2002, 2003, 2007). Additional findings suggest that the N350 is larger for more complex images (Ruchkin, Johnson, Canoune, \& Ritter, 1991; Schendan \& Kutas, 2002; Stuss, Sarazin, Leech, \& Picton, 1983 ) as well as for non-typical image views relative to easier to recognize, canonical views, consistent with the idea that the 
N350 effect is related to the selection of a stored structural description model to match against the perceptual input (Schendan \& Kutas, 2003). Our results are consistent with previous research on the N350 effect and show that color information is activated in the N350 time window. This, together with the fact that subjects were faster in verifying typical color objects, suggests that shape and color effects are combined to facilitate the selection of structural descriptions from the long-term memory in this time window. The fact that we did not observed surface color effects in early ERP components corroborates the account that color information is activated together with the structural description. In this context, the typical surface color might limit the possible structural descriptions that match with the presented object in the early part of the identification process. In addition, for the non-matching trials in the color knowledge verification task, we observed an effect post-500 ms (referred to as LPC), with a typical frontal topography (Hanslmayr et al., 2008; Liotti, Woldorff, Perez, \& Mayberg, 2000). This shows that atypical color objects were associated with a more negative ERP over the frontal sites during the time window between 580 and $720 \mathrm{~ms}$ after stimulus onset. Previous ERP studies suggest that the LPC effect is related to activation of associated semantic knowledge (see, for example, Mazerolle, D'Arcy, Marchand, \& Bolster, 2007). Similarly to the N350 effect, the LPC effect varies with recognition success; however, unlike the N350, the LPC shows categorization modulations for any image, regardless of recoverability. The LPC may index a fronto-parietal network for categorization-related processes, such as selection of an appropriate response, including a name (Schendan \& Kutas, 2002, 2003, 2007). Although LPC repetition effects could reflect memory for these later categorization processes, most evidence suggest that these effects reflect conscious recollection (Duarte, Ranganath, Winward, Hayward, \& Knight, 2004; Paller, Kutas, \& McIsaac, 1995). The color effects found in this ERP component suggest that typical color facilitates the activation of semantic object knowledge. Taken together, the LPC and N350 effects that we observed suggest that object color helps in the identification process, by facilitating access to the structural description and associated semantic knowledge about the object. The behavioral results are consistent with this idea: subjects were faster in responding to the typical color objects in both matching and non-matching trials. Additionally, the matching effect in the color knowledge verification task showed that non-matching trials were associated with a more negative potential over the central channels in a time window between 350 and $600 \mathrm{~ms}$ after stimulus onset, consistent with an N400-like effect. This significant N400-like effect was found for both typical and atypical color objects, suggesting that semantic knowledge about the object color was activated during the task, even when the objects were presented in a non-typical color (e.g., a strawberry painted in gray). Whenever the color knowledge activated by the object did not match with the previous presented color name (non-matching trial), a greater N400 was observed. This result is consistent with previous literature showing N400 effects when a picture is semantically anomalous in a given context (see, for example, Hamm et al., 2002).

In the surface verification task, we did not find any significant effects related to color knowledge (neither behavioral nor ERP effects). Subjects verified equally fast and use the same cognitive resources to determine that a red strawberry (a typical red object) and a red mouse (a typical non-red object) are colored red, and that a gray strawberry and a gray mouse are not colored in red. Thus, we suggest that when color knowledge is not necessary to resolve a task, this information is not automatically activated. An alternative interpretation is that subjects performed the surface verification task before the object color knowledge was activated, or possibly that the task was too easy and did not implicate explicit object recognition to accurately perform the task. In other words, subjects could have decided about the surface color without completing the identification of the object and without a full activation of the object properties and features. This set of circumstances might explain the absence of effects that were present in the behavioral results; however, they cannot explain the absence of ERP effects. It is well documented that $\sim 200-300 \mathrm{~ms}$ after stimuli onset, functional and perceptual properties of the objects are automatically activated (Vihla, Laine, \& Salmelin, 2006). Thus a better explanation of our results is that color knowledge was activated conditionally during the surface color verification task. Nevertheless, we believe that it is important to replicate this result with a more complex verification task, where explicit object identification is necessary to accurately perform the task. Furthermore, we observed that non-matching trials in the surface color verification task were associated with a more negative potential over the frontal sites $\sim 200 \mathrm{~ms}$ after stimulus onset and with a more central negative potential $\sim 300 \mathrm{~ms}$ after stimuli onset, compared with the matching trials. This negative frontal potential shares some properties with the N2 component, with regard to both latency and topographic distribution (Folstein \& Petten, 2008). N2 effects have been shown in conditions that require inhibition of a prepared response and/or contain elements suggesting two conflicting responses, as compared to conditions without response inhibition or response conflict (Nieuwenhuis, Yeung, Wildenberg, \& Ridderinkhof, 2003; Pfefferbaum, Ford, Weller, \& Kopell, 1985). Folstein and collaborators (2008) suggested that enhancement of the N2 is due to conflicting information that occur when participants begin to prepare a motor response before evaluation of a stimulus is complete. This hypothesis suggests that the N2 effect is sensitive to response conflict (Folstein, Van Petten, \& Rose, 2008). In accordance with this idea, we suggest that the N2 effect found in our study is related with motor-related preparation, and whenever an object was colored in a different color from the previously presented color name (non-matching trial), a stronger N2 component was elicited.

At the neuroanatomical level, distinct neural regions appear to be differentially engaged during the processes of color perception and the retrieval of object color knowledge. Whereas color perception is more closely associated with the occipital and posterior occipitotemporal cortex (Bartels \& Zeki, 2000; Chao \& Martin, 1999; Zeki \& Marini, 1998), color knowledge is associated with the left anterior inferior temporal, left frontal and left superior parietal regions of the brain (Chao \& Martin, 1999; Wiggs, Weisberg, \& Martin, 1999). Also, neuropsychological studies have reported dissociation between surface color and color knowledge in the ventral occipitotemporal cortex. Whereas lesions in the lingual gyrus result in achromatopsia in the presence of spared color knowledge (Bouvier \& Engel, 2006), lesions in the ventral temporal cortex results in color agnosia with spared color perception (Miceli et al., 2001). Together, these studies suggest that the brain regions engaged during the retrieval of object-color knowledge are different from those areas engaged during color perception. However, the dissociation between perception and knowledge retrieval mechanisms does not necessarily imply that these two abilities are independent. For example, some neuroimaging results suggest that color knowledge modulates regions that are involved in color perception (Goldberg, Perfetti, \& Schneider, 2006; Howard et al., 1998; Kellenbach, Brett, \& Patterson, 2001; Simmons et al., 2007; Ueno et al., 2007). Previous studies have investigated color perception with the ERP technique (Anllo-Vento, Luck, \& Hillyard, 1998; Buchner, Weyer, Frackowiak, Romaya, \& Zeki, 1994; Edwards, Xiao, Keysers, Földiák, \& Perrett, 2003; Goffaux et al., 2005; Lu et al., 2010; Plendl et al., 1993; Proverbio, Burco, Zotto, \& Zani, 2004). For instance, Goffaux and colleagues (2005) measured early ERPs to examine the effect of color cues on scene categorization. The ERPs associated with the black and white images and with the 
atypical colored scenes were delayed compared to the ones associated with the typical colored scenes. The color effects were mirrored in the early (150 ms following stimulus onset) frontal EEG correlates.

Overall, the objective of the present study was to explore the interactions between top-down color knowledge and bottom-up color perception during object recognition. Our study provides electrophysiological evidence suggesting that surface color input and color knowledge retrieval interact during object recognition. The results suggest that the appropriate surface color prompts the activation of color knowledge, thereby facilitating object recognition. Although color knowledge could be activated without the presence of color input, the surface color input triggers the activation of stored color knowledge, contributing to more efficient object identification, both facilitating the selection of a stored object representation from long-term memory and activating the associated semantic object knowledge.

\section{Acknowledgments}

This work was supported by Fundação para a Ciência e Tecnologia (REEQ/879/PSI/2005; PTDC/PSI-PCO/110734/2009; IBB/CBME, LA, FEDER/POCI 2010), Max Planck Institute for Psycholinguistics, Donders Institute for Brain, Cognition and Behaviour, Radboud University Nijmegen, Vetenskapsrådet (8276), Hedlunds Stiftelse and Stockholm County Council (ALF, FoUU). Inês Bramão was supported by a PhD fellowship (FCT/SFRH/BD/27381/2006).

\section{Appendix A. Stimuli used in experiment}

\begin{tabular}{ll}
\hline Color & Object name \\
\hline Red & $\begin{array}{l}\text { Apple, Cherry, Heart, Lips, Strawberry, Tomato, } \\
\text { Ladybug }\end{array}$ \\
Gray & $\begin{array}{l}\text { Key, Mouse, Nail, Scissors, Hippopotamus, Shark, } \\
\text { Elephant }\end{array}$ \\
Orange & $\begin{array}{l}\text { Carrot, Lobster, Orange, Pineapple, Pumpkin, Crab, } \\
\text { Traffic cone }\end{array}$ \\
Green & Alligator, Artichoke, Frog, Lettuce, Pepper, Tree, Pea \\
Yellow & Banana, Bee, Lemon, Star, Sun, Cheese, Bird \\
Brown & Camel, Deer, Fox, Kangaroo, Monkey, Peanut, Nut \\
Pink & Arm, Ear, Finger, Pig, Leg, Hand, Foot \\
White & Cigarette, Cloud, Sheep, Swan, Bone, Igloo, Tooth \\
\hline
\end{tabular}

\section{References}

Anllo-Vento, L., Luck, S., \& Hillyard, S. (1998). Spatio-temporal dynamics of attention to color: Evidence from human electrophysiology. Human Brain Mapping, 6, 216-238.

Barrett, S., \& Rugg, M. (1990). Event-related potentials and the semantic matching of pictures. Brain and Cognition, 14, 201-212.

Bartels, A., \& Zeki, S. (2000). The architecture of the colour centre in the human visual brain: New results and a review. European Journal of Neuroscience, 12 $172-193$.

Bouvier, S., \& Engel, S. (2006). Behavioral deficits and cortical damage loci in cerebral achromatopsia. Cerebral Cortex, 16, 183-191.

Bramão, I., Faísca, L., Petersson, K. M., \& Reis, A. (2010). The influence of surface color information and color knowledge information in object recognition. American Journal of Psychology, 123, 459-468.

Buchner, H., Weyer, U., Frackowiak, R., Romaya, J., \& Zeki, S. (1994). The timing of visual evoked potential activity in human area V4. Proceedings of the Royal Society of London, B, 257, 99-104.

Chao, L., \& Martin, A. (1999). Cortical regions associated with perceiving, naming, and knowing about colors. Journal of Cognitive Neuroscience, 11, 25-35.

Clark, H. (1973). The language-as-fixed-effect fallacy: A critique of language statistics in psychological research. Journal of Verbal Learning and Verbal Behaviour, 12, 335-359.
Duarte, A., Ranganath, C., Winward, L., Hayward, D., \& Knight, R. T. (2004). Dissociable neural correlates for familiarity and recollection during the encoding and retrieval of pictures. Cognitive Brain Research, 18, 255-272.

Edwards, R., Xiao, D., Keysers, C., Földiák, P., \& Perrett, D. (2003). Color sensitivity of cells responsive to complex stimuli in the temporal cortex. Journal of Neurophysiology, 90, 1245-1256.

Folstein, J., \& Petten, C. (2008). Influence of cognitive control and mismatch on the N2 component of the ERP: A review. Psychophysiology, 45, 152-170.

Folstein, J., Van Petten, C., \& Rose, S. A. (2008). Novelty and conflict in the categorization of complex stimuli. Psychophysiology, 45, 467-479.

Ganis, G., Kutas, M., \& Sereno, M. (1996). The search for "common sense": An electrophysiological study of comprehension of words and pictures in reading. Journal of Cognitive Neuroscience, 8, 89-106.

Goffaux, V., Jacques, C., Mouraux, A., Oliva, A., Schyns, P., \& Rossion, B. (2005). Diagnostic colours contribute to the early stages of scene categorization: Behavioural and neurophysiological evidence. Visual Cognition, 12, 878-892.

Goldberg, P., Perfetti, C., \& Schneider, W. (2006). Perceptual knowledge retrieval activates sensory brain regions. The Journal of Neuroscience, 26, 4917-4921.

Hamm, J., Johnson, B., \& Kirk, I. (2002). Comparison of the N300 and N400 ERPs to picture stimuli in congruent and incongruent contexts. Clinical Neurophysiology, 113, 1339-1350.

Hanslmayr, S., Pastötter, B., Bäuml, K., Gruber, S., Wimber, M., \& Klimesch, W. (2008). The electrophysiological dynamics of interference during the Stroop task. Journal of Cognitive Neuroscience, 20, 215-225.

Holcomb, P., \& McPherson, W. (1994). Event-related brain potentials reflects semantic priming in an object decision task. Brain and Cognition, 24, 259-276.

Howard, R., Ffytche, D., Barnes, J., McKeefry, D., Ha, Y., Woodruff, P., et al. (1998). The functional anatomy of imagining and perceiving colour. NeuroReport, 9 1019-1023.

Joseph, J. (1997). Color processing in object verification. Acta Psychologica, 97, 95-127.

Joseph, J., \& Proffitt, D. (1996). Semantic versus perceptual influences of color in object recognition. Journal of Experimental Psychology: Learning, Memory, and Cognition, 22, 407-429.

Kellenbach, M., Brett, M., \& Patterson, K. (2001). Large, colorful, or noisy? Attributeand modality-specific activations during retrieval of perceptual attribute knowledge. Cognitive, Affective, \& Behavioral Neuroscience, 1, 207-221.

Kutas, M., \& Hillyard, S. (1980a). Event-related potentials to semantically inappropriate and surprisingly large words. Biological Psychology, 11, 99-116.

Kutas, M., \& Hillyard, S. (1980b). Reading senseless sentences: Brain potentials reflect semantic incongruity. Science, 207, 203-205.

Liotti, M., Woldorff, M. G., Perez, R., \& Mayberg, H. S. (2000). An ERP study of the temporal course of the Stroop color-word interference task. Neuropsychologia, $38,701-711$.

Lu, A., Xu, G., Jin, H., Mo, L., Zhang, J., \& Zhang, J. X. (2010). Electrophysiological evidence for effects of color knowledge in object recognition. Neuroscience Letters, 469, 405-410.

Maris, E. (2004). Randomization tests for ERP-topographies and whole spatiotemporal data matrices. Psychophysiology, 41, 142-151.

Maris, E., \& Oostenveld, R. (2007). Nonparametric statistical testing of EEG- and MEG-data. Journal of Neuroscience Methods, 164, 177-190.

Mazerolle, E. L., D’Arcy, R. C. N., Marchand, Y., \& Bolster, R. B. (2007). ERP assessment of functional status in the temporal lobe: Examining spatiotemporal correlates of object recognition. International Journal of Psychophysiology, 66, 81-92.

McPherson, W., \& Holcomb, P. (1999). An electrophysiological investigation of semantic priming with pictures of real objects. Psychophysiology, 36, 53-65.

Miceli, G., Fouch, E., Capasso, R., Shelton, J. R., Tomaiuolo, F., \& Caramazza, A. (2001). The dissociation of color from form and function knowledge. Nature Neuroscience, 4, 664-667.

Nieuwenhuis, S., Yeung, N., Wildenberg, W., \& Ridderinkhof, R. (2003) Electrophysiological correlates of anterior cingulate function in a go/nogo task: Effects of response conflict and trial type frequency. Cognitive, Affective, $\mathcal{E}$ Behavioral Neuroscience, 3, 17-26.

Nigam, A., Hoffman, J., \& Simons, R. (1992). N400 to semantically anomalous pictures and words. Journal of Cognitive Neuroscience, 4, 15-22.

Oostenveld, R., Fries, P., \& Jensen, O. (2009). Fieldtrip toolbox. <http://www.ru.nl/ fcdonders/fieldtrip>.

Paller, K. A., Kutas, M., \& McIsaac, H. K. (1995). Monitoring conscious recollection via the electrical activity of the brain. Psychological Science, 6, 107-111.

Pfefferbaum, A., Ford, J. M., Weller, B. J., \& Kopell, B. S. (1985). ERPs to response production and inhibition. Electroencephalography and Clinical Neurophysiology, $60,423-434$

Pietrowsky, R., Kuhmann, W., Krug, R., Mölle, M., Fehm, H., \& Born, J. (1996). Eventrelated brain potentials during identification of tachistoscopically presented pictures. Brain and Cognition, 32, 416-428.

Plendl, H., Paulus, W., Roberts, I. G., Bõtzel, K., Towell, A., Pitman, J. R., et al. (1993). The time course and location of cerebral evoked activity associated with the processing of colour stimuli in man. Neuroscience Letters, 150, 9-12.

Pratarelli, M. (1994). Semantic processing of pictures and spoken words: Evidence from event-related brain potentials. Brain and Cognition, 24, 137-157.

Proverbio, A. M., Burco, F., Zotto, M., \& Zani, A. (2004). Blue piglets? Electrophysiological evidence for the primacy of shape over color in object recognition. Cognitive Brain Research, 18, 288-300.

Raaijmakers, J. (2003). A further look at the 'Language-as-fixed-effect fallacy'. Canadian Journal of Experimental Psychology, 57, 141-151. 
Raaijmakers, J., Schrijnemakers, J., \& Gremmen, F. (1999). How to deal with 'The language-as-fixed-effect fallacy': Common misconceptions and alternative solutions. Journal of Memory and Language, 41, 416-426.

Rossion, B., \& Pourtois, G. (2004). Revisiting Snodgrass and Vanderwart's object pictorial set: The role of surface detail in basic-level object recognition. Perception, 33, 217-236.

Ruchkin, D. S., Johnson, R., Canoune, H., \& Ritter, W. (1991). Event-related potentials during arithmetic and mental rotation. Electroencephalography and Clinical Neurophysiology, 79, 473-487.

Schendan, H., \& Kutas, M. (2002). Neurophysiological evidence for two processing times for visual object identification. Neuropsychologia, 40, 931-945.

Schendan, H., \& Kutas, M. (2003). Time course of processes and representations supporting visual object identification and memory. Journal of Cognitive Neuroscience, 15, 111-135.

Schendan, H., \& Kutas, M. (2007). Neurophysiological evidence for the time course of activation of global shape, part, and local contour representations during visual object categorization and memory. Journal of Cognitive Neuroscience, 19, 734-749.

Schutter, D., Leitner, C., Kenemans, J. L., \& van Honk, J. (2006). Electrophysiological correlates of cortico-subcortical interaction: A cross-frequency spectral EEC analysis. Clinical Neurophysiology, 117, 381-387.

Simmons, W., Ramjee, V., Beauchamp, M., McRae, K., Martin, A., \& Barsalou, L. (2007). A common neural substrate for perceiving and knowing about color. Neuropsychologia, 45, 2802-2810.
Snodgrass, J. G., \& Vanderwart, M. (1980). A standardized set of 260 pictures: Norms for name agreement, image agreement, familiarity, and visual complexity. Journal of Experimental Psychology: Learning, Memory and Cognition, 6, 174-215.

Stuss, D. T., Picton, T. W., Cerri, A. M., Leech, E. E., \& Stethem, L. L. (1992). Perceptual closure and object identification: Electrophysiological responses to incomplete pictures. Brain and Cognition, 19, 253-266.

Stuss, D. T., Sarazin, F. F., Leech, E. E., \& Picton, T. W. (1983). Event-related potentials during naming and mental rotation. Electroencephalography and Clinical Neurophysiology, 56, 133-146.

Tanaka, J., \& Presnell, L. (1999). Color diagnosticity in object recognition. Perception E Psychophysics, 61, 1140-1153.

Tanaka, J., Weiskopf, D., \& Williams, P. (2001). The role of color in high-level vision. Trends in Cognitive Sciences, 5, 211-215.

Ueno, A., Abe, N., Suzuki, M., Hirayama, K., Mori, E., Tashiro, M., et al. (2007). Reactivation of medial temporal lobe and occipital lobe during the retrieval of color information: A positron emission tomography study. NeuroImage, 34, 1292-1298.

Vihla, M., Laine, M., \& Salmelin, R. (2006). Cortical dynamics of visual/semantic vs. phonological analysis in picture confrontation. NeuroImage, 33, 732-738.

Wiggs, C., Weisberg, J., \& Martin, A. (1999). Neural correlates of semantic and episodic memory retrieval. Neuropsychologia, 37, 103-118.

Zeki, S., \& Marini, L. (1998). Three cortical stages of colour processing in the human brain. Brain, 121, 1669-1685. 